\title{
A Letter in Support of Real-World RECIST
}

Ajeet Gajra · Bruce A. Feinberg

Received: January 9, 2020 / Published online: February 13, 2020

(C) The Author(s) 2020

Keywords: EHR; Electronic health record; Realworld data; RECIST criteria; Tumor response assessment

\section{Dear Editor,}

We read with interest the report "Generating real-world tumor burden endpoints from electronic health record data: comparison of RECIST, radiology-anchored, and clinician-anchored approaches for abstracting real-world progression in non-small cell lung cancer" by Griffith et al. in Advances in Therapy [1]. We compliment the authors on their work to evaluate surrogates for tumor response assessment in real-world data (RWD). However, we would like to extend caution against the inference that RECIST is not a feasible modality for response assessment in RWD and the corollary inference that a surrogate response measure is needed for RWD. The reasons for such caution are threefold: Firstly, our group has demonstrated, across

Letter to the editor in response to the article entitled "Generating real-world tumor burden endpoints from electronic health record data: comparison of RECIST, radiology-anchored, and clinician-anchored approaches for abstracting real-world progression in non-small cell lung cancer" by Griffith et al. [1].

A. Gajra $(\bowtie) \cdot$ B. A. Feinberg

Cardinal Health, Dublin, OH, USA

e-mail: Ajeet.Gajra@Cardinalhealth.com a variety of tumor types, in over 800 unique patients that RECIST can be applied retrospectively in the real-world setting [2-5]. Second, this report tends to confuse the issue of a standardized radiologic response "radiology-anchored" (as measured by RECIST) with "clinician-anchored" response in which the assessment is not only radiologic but also may incorporate patient history (symptoms, performance status), physical examination, biomarkers, and adverse events among other criteria. The clinician-anchored response may paint a more complete picture of the patient's overall status as compared to the radiology-anchored RECIST-based scan report but it cannot be correlated with published response as obtained through randomized controlled trials (RCT). Third, we seek clarification from the authors regarding the differences between the radiology-anchored and RECIST-based approaches. Since both RECIST and radiology-anchored approaches would require a detailed evaluation of the radiology report then is it safe to assume that the RECIST-based approach should have provided the same information on real-world progression as the radiology-anchored approach, even in the absence of detailed RECIST criteria.

There do exist barriers to routinely incorporating RECIST in routine practice but none of them are insurmountable. Radiologists do not routinely provide RECIST measurements for all 
scans requiring tumor response assessment in the real-world setting. Potential reasons for such a lack of RECIST-based reporting by the radiologists include a dearth of time on the part of the radiologist, a lack of detailed knowledge of RECIST reporting, and/or a lack of awareness of the need or importance of RECIST-based reporting on any given scan. The last of these may in part be contributed by the requesting oncology clinicians who do not always ask for target lesion assessment or provide adequate history or context on the requisitions to the radiologists. Another barrier that can impact both oncology clinicians and radiologists is the use different radiology facilities (which do not communicate easily with each other digitally) leading to the lack of availability of prior films for comparison. Hence, it is not surprising that a sample of randomly chosen retrospective imaging reports yielded low levels of RECISTspecific data. Notably 58\% did have "radiology reports appropriate for RECIST assessment". These finding should not be misconstrued as a RECIST-based approach being infeasible in explorations of RWD. In fact, given that various other concessions are unavoidable (e.g., using surrogates such as time to next treatment for progression-free survival), when assessing response in RWD, progression does not have to be one.

RECIST criteria are well validated and essentially considered the gold standard for tumor response assessment. They are updated by a committee of global experts and modifications are applied on the basis of evidence relevant to specific populations, e.g., iRECIST for patient treated with immunotherapy.

We suggest a threefold plan:

1. Utilize available technology to obtain RECIST retrospectively by a review of actual films.

(a) PACS (picture archiving and communication system) is a medical imaging technology used primarily in healthcare organizations to securely store and digitally transmit electronic images and clinically relevant reports. These images are accessible to clinicians and radiologists for assessment and measurements at virtually every US hospital. Physician abstractors can access digital images to provide RECIST measurements, if not included in radiology reports, the method we employed in our real-world RECIST research referenced above.

(b) MIM is an FDA (US Food and Drug Administration)-approved application for sharing radiology images on mobile platforms. The app enables clinicians to measure distance, intensity values, and display measurement lines, annotations, and regions of interest. The images are securely transferred to the app from a hospital or physician's office through a secure network transfer facilitated by MIM.

(c) Use artificial intelligence to derive outcomes from radiology reports. The feasibility of ascertaining oncologic outcomes from radiology reports has recently been demonstrated using deep natural language processing [6]. Such technology can dramatically alter the field especially if measurements are included within radiology reports.

2. Create a workstream within electronic health record (EHR) systems such as Flatiron to request RECIST-based assessment in radiology requisitions.

3. Train radiologists in RECIST. Ideally, if radiologists were to report RECIST on every scan performed for tumor response assessment then the task of RW response assessment would be remarkably simplified. There is a need for diagnostic radiologists to specialize in such oncoradiology evaluation akin to other areas such as neuroimaging, skeletal imaging, mammography, etc. Such training is offered at very limited institutions at the present time. Such radiologic oncologists will be able to serve the needs of patients with cancer by collaborating with their medical, surgical, and radiation oncologist colleagues.

Although we applaud the investigators for their efforts, questions regarding their methodology raise concern, e.g., were the 26 patients in 
experiment 1 a subset of the 200 in experiment 2 and, if not, what is the explanation for this distinction? Methodological issues as well as challenges to their assumptions regarding the inability to conduct retrospective RECIST on RWD gave us pause to present solutions that will allow direct comparisons between RWD response assessments and those from RCT.

\section{ACKNOWLEDGEMENTS}

Funding. No funding or sponsorship was received for this study or publication of this letter.

Authorship. All named authors meet the International Committee of Medical Journal Editors (ICMJE) criteria for authorship for this article, take responsibility for the integrity of the work as a whole, and have given their approval for this version to be published.

Disclosures. Ajeet Gajra: Employment with Cardinal Health and ICON clinical research. Bruce Feinberg: Employment with Cardinal Health.

Compliance with Ethics Guidelines. This letter is based on previously conducted studies and does not contain any studies with human participants or animals performed by any of the authors.

Peer Review. Please note, contrary to the journal's standard single-blind peer-review process, as a letter this article underwent review by a member of the journal's Editorial Board.

Open Access. This article is licensed under a Creative Commons Attribution-NonCommercial 4.0 International License, which permits any non-commercial use, sharing, adaptation, distribution and reproduction in any medium or format, as long as you give appropriate credit to the original author(s) and the source, provide a link to the Creative Commons licence, and indicate if changes were made. The images or other third party material in this article are included in the article's Creative Commons licence, unless indicated otherwise in a credit line to the material. If material is not included in the article's Creative Commons licence and your intended use is not permitted by statutory regulation or exceeds the permitted use, you will need to obtain permission directly from the copyright holder. To view a copy of this licence, visit http://creativecommons.org/licenses/by$\mathrm{nc} / 4.0 /$.

\section{REFERENCES}

1. Griffith SD, Tucker M, Bowser B, et al. Generating real-world tumor burden endpoints from electronic health record data: comparison of RECIST, radiologyanchored, and clinician-anchored approaches for abstracting real-world progression in non-small cell lung cancer. Adv Ther. 2019;36(8):2122-36.

2. Feinberg BA, Bharmal M, Klink AJ, et al. Using response evaluation criteria in solid tumors in realworld evidence cancer research. Future Oncol. 2018;14(27):2841-8.

3. Luke JJ, Ghate SR, Kish J, et al. Targeted agents or immuno-oncology therapies as first-line therapy for BRAF-mutated metastatic melanoma: a real-world study. Future Oncol. 2019;15(25):2933-42.

4. Chatterjee D, Kish J, Knoth R, et al. Real-world outcomes with multiple second-line treatments after first-line lenvatinib in patients with radioactiveiodine refractory differentiated thyroid cancer (RAIR DTC). Thyroid. 2019;P-1-A-166 [Abstr 388].

5. Mougalian SS, Feinberg BA, Wang E, Alexis K, Chatterjee D, Knoth RL, Nero D, Miller T, Liassou D, Kish JK. Observational study of clinical outcomes of eribulin mesylate in metastatic breast cancer after cyclin-dependent kinase 4/6 inhibitor therapy. Future Oncol. 2019;15(34):3935-44.

6. Kehl KL, Elmarakeby H, Nishino M, et al. Assessment of deep natural language processing in ascertaining oncologic outcomes from radiology reports. JAMA Oncol. 2019. https://doi.org/10.1001/jamaoncol. 2019.1800. 